\title{
Aplicação da metodologia coaching para o tratamento da obesidade: uma visão multidisciplinar
}

\author{
Application of coaching methodology to obesity treatment: \\ a multidisciplinary view
}

\author{
Fabiano Porto Mendes ${ }^{1}$ \\ Norberto da Cunha Garin ${ }^{2}$ \\ Edgar Zanini Timm ${ }^{3}$ \\ Jerri Luiz Ribeiro ${ }^{4}$
}

\section{RESUMO}

A metodologia coaching é utilizada para o desenvolvimento de pessoas nas dimensões profissional e pessoal, valendo-se do apoio ao processo de reflexão, aprendizado e ação para alcançar metas. Objetivo: estudar a aplicação da metodologia coaching no tratamento da obesidade. Metodologia: estudou-se dificuldades para o tratamento da obesidade e possíveis estratégias de tratamento através desta metodologia. Resultados: (1) a obesidade atualmente é problema de saúde pública considerado uma epidemia mundial, sendo uma doença multifatorial; (2) o obeso tem pressa para emagrecer e a maior dificuldade está em manter sua motivação para continuar o tratamento em médio e longo prazo; (3) o coaching pode fazer parte de um programa multidisciplinar, atuando fortemente na mudança comportamental. Conclusão: a metodologia coaching está sendo utilizada com êxito por profissionais de diferentes dimensões da área da saúde, podendo, como ficou claro, ser adaptada conforme a prática do profissional que a utiliza no tratamento da obesidade.

\section{PALAVRAS-CHAVE}

Metodologia coaching; Tratamento da obesidade; Saúde Humana.

\footnotetext{
'Licenciado em Educação Física (ULBRA), Especialista em Treinamento Desportivo e em Administração e Marketing Esportivo (UGF), Mestre em Reabilitação e Inclusão (IPA), Professor de Lutas, Personal Trainer e Coach na Companhia Athletica Porto Alegre, Porto Alegre/RS.

2Bacharel em Teologia (FT/IMS), Licenciado em Filosofia (UPF), Mestre em Teologia (EST), Doutor em Teologia (EST), docente e pesquisador no PPG em Reabilitação e Inclusão do Centro Universitário Metodista - IPA, Porto Alegre/RS.

${ }^{3}$ Licenciado em Educação Física (UFPel), Licenciado em Filosofia (PUCRS), Mestre em Educação (PUCRS), Doutor em Educação (PUCRS), docente e pesquisador no PPG em Reabilitação e Inclusão do Centro Universitário Metodista -IPA, Porto Alegre/RS. ${ }^{4}$ Licenciado em Educação Física (UFRGS), Doutor em Ciências do Movimento Humano (UFRGS), docente e pesquisador no PPG em Reabilitação e Inclusão e no PPG em Biociências e Reabilitação do Centro Universitário Metodista - IPA, Porto Alegre/RS.
} 


\section{ABSTRACT}

The coaching methodology is used for the development of people in professional and personal dimensions, drawing on support for the process of reflection, learning and action to achieve goals. Objective: To study the application of coaching methodology in the treatment of obesity. Methodology: it was studied difficulties for the treatment of obesity and possible treatment strategies using this methodology. Results: (1) obesity is currently considered a public health problem worldwide epidemic, is a multifactorial disease; (2) the obese to lose weight in a hurry and the biggest difficulty lies in keeping your motivation to continue treatment in the medium and long term; (3) coaching can be part of a multidisciplinary program, strongly acting on behavioral change. Conclusion: The coaching methodology is being used successfully by professionals from different dimensions of health, and may, as is clear, be adapted according to the practice of professional uses in the treatment of obesity.

\section{KEYWORDS}

Coaching methodology; Treatment of obesity; Health-slimming. 


\section{INTRODUÇÃO}

A obesidade tem se mostrado um problema de saúde pública acentuado na contemporaneidade, alcançando grande parte da população mundial. De acordo com a Organização Mundial da Saúde (OMS) a prevalência de sobrepeso em 2006 era de 1,2 biIhões e de obesos 400 milhões. Estima-se que em 2016, o número de sobrepeso aumente para 1,6 biIhões e que mais de 700 milhões sejam obesos ${ }^{1}$. O Instituto Brasileiro de Geografia e Estatística (IBGE) na pesquisa feita em 2009 afirma que obesidade é uma epidemia em muitas nações ocidentais, incluindo o Brasil, onde há cerca de 17 milhões de obesos, o que representa $9,6 \%$ da população brasileira. Dados de pesquisas brasileiras mostram que há um aumento marcante do excesso de peso (sobrepeso) entre os brasileiros, chegando a atingir quase $50 \%$ dos adultos conforme o IBGE${ }^{2}$. Nesse levantamento, foi encontrada uma prevalência de obesidade de 16,9\% entre as mulheres e $12,5 \%$ entre os homens ${ }^{2}$.

$\mathrm{A} \mathrm{OMS}^{3}$, define obesidade como um excesso de gordura corporal acumulada no tecido adiposo, com implicações para a saúde, sendo uma condição complexa de dimensões sociais, biológicas e psicossociais consideráveis, podendo eventualmente afetar qualquer pessoa, independentemente da idade ou do grupo socioeconômico, em qualquer parte do mundo.

O grande desafio no combate a obesidade parece ser a adesão ao tratamento ${ }^{4}$. Conforme Boas ${ }^{5}$, a adesão ao autocuidado é definida como a extensão na qual o comportamento da pessoa se refere ao uso de medicação, ao seguimento de dietas e a prática diária de atividades físicas para o favorecimento da mudança de comportamento e adoção de hábitos de vida saudáveis. O Coaching pode ser uma ferramenta muito eficaz para profissionais que buscam múltiplas habilidades e competências além daquelas de sua formação profissional básica, para aumentar a probabilidade de adesão ao tratamento da obesidade.

Segundo o entendimento de Paiva ${ }^{6}$, Coaching é um processo que visa aumentar o desempenho de um indivíduo (grupo ou empresa), aumentando os resultados positivos, através de metodologias, ferramentas e técnicas conduzidas por um profissional (Coach) em uma parceria sinérgica e dinâmica com o cliente (Co- achee). Trata-se de uma metodologia que proporciona expansão significativa da performance profissional e produtividade pessoal: coaching implica como sair de um ponto (estado atual) e chegar a outro ponto (estado desejado). A essência do Coaching consiste no apoio a uma pessoa, grupo ou empresa na promoção da mudança que deseja, rumo a uma nova direção ou a um novo estado desejado.

Para Carvalho ${ }^{7}$ o Coaching pode ser um aliado àqueles que desejam perder ou manter o peso, pois conduz a pessoa a mudanças de hábitos sabotadores que a impedem de levar uma vida saudável e adquirir comportamentos que auxiliarão na redução e manutenção de peso. O processo auxiliará o Coachee (cliente) a analisar questões que influenciam na conquista do seu objetivo: a perda de peso. Fatores como gestão de tempo, disciplina, comprometimento, crenças, valores e motivação são trabalhados durantes as sessões. No Coaching são desenvolvidos planos de ação para alcançar objetivos, onde o próprio indivíduo traçará metas a serem cumpridas diariamente e, através disso, adquirirá disciplina e foco.

Tendo em vista a problemática para o tratamento da obesidade em adultos jovens é possível perguntar pela possibilidade da aplicação ressignificada dos conceitos da metodologia coaching no tratamento da obesidade. Assim a pesquisa que realizamos problematizou a aplicação da metodologia coaching no tratamento da obesidade, estudando diferentes concepções e modos de prática por profissionais da saúde. Com essa intenção, buscou-se: (a) analisar o problema obesidade atualmente; (b) estudar as dificuldades para o tratamento da obesidade; e (c) identificar possíveis estratégias de tratamento para obesidade através da metodologia coaching.

A seguir apresenta-se o método adotado para o desenvolvimento dos estudos em tela.

\section{MATERIAL E MÉTODOS}

A pesquisa caracterizou-se como estudos de natureza qualitativa. $\mathrm{O}$ conjunto das pessoas que participaram foi formado por seis profissionais que atuam na área da saúde, sendo um médico (MD), um psicólogo (PS), um nutricionista (NT), um educador físico - personal trainer- (PT), um assistente social 
(AS) e um master coach (MC); tendo sido entrevistadas quatro mulheres e dois homens. Todos os participantes possuem formação superior, inclusive com estudos em nível de pós graduação; sendo um deles com doutorado. Os participantes trabalham com o tratamento da obesidade e utilizam a metodologia coaching em seus processos de trabalho, porém, apenas um deles não tem formação específica em coaching, entretanto tem formação terapêutica e utiliza ferramentas do método coaching.

Cada um dos participantes, a partir dos seus conhecimentos teóricos e de prática profissional com o coaching, respondeu a uma entrevista semiestruturada, via email. Os dados coletados foram sistematizados e trabalhados com a técnica de análise de conteúdo fundamentada em Bardin .

\section{RESULTADOS E DISCUSSÃO}

O conhecimento construído na pesquisa está sistematizado nos seguintes pontos: (1) o problema obesidade; (2) o tratamento da obesidade; (3) dificuldades no tratamento da obesidade; (4) coaching no tratamento da obesidade; (5) aplicação da metodologia coaching no tratamento da obesidade.

\section{O problema obesidade}

A pesquisa evidenciou a percepção dos profissionais da saúde entrevistados sobre o problema da obesidade na contemporaneidade, em seus aspectos conceituais, sociais, nutricionais, de atividade física, e emocionais. No que tange a parte conceitual da obesidade, PT ressaltou que a obesidade é "um dos maiores problemas de saúde pública nos dias de hoje. Uma doença crônica que está se tornando uma epidemia em todo o mundo". Nesta mesma linha de conceituação, NT compartilha da mesma opinião, quando relata que "a obesidade nos dias de hoje é um problema de Saúde Pública". Ressaltando essa questão epidêmica da obesidade o $\mathrm{IBGE}^{2}$ em pesquisa feita em 2009, afirma que obesidade é uma epidemia em muitas nações ocidentais, incluindo o Brasil, onde há cerca de 17 milhões de obesos, o que representa $9,6 \%$ da população brasileira. Dentro dos principais motivos que levam a obesidade destacam-se os fatores sociais, pois a vida moderna pode con- duzir as pessoas à má alimentação e à falta de tempo, sendo neste ponto, novamente ressaltado o caráter nutricional. PT relatou: "a vida agitada que as pessoas levam atualmente, resultando em falta de tempo, estresse e ansiedade, bem como, questões econômicas e culturais influenciam no aumento da obesidade". Nessa mesma linha de raciocínio de PT pode-se juntar a análise de NT quando declara: "devido à correria do dia-a-dia as pessoas optam por alimentos produzidos rapidamente" os quais nem sempre são saudáveis e contribuem para obesidade. MD concordou com essa opinião ao afirmar: "A obesidade é decorrente do estilo de vida atual". MC fez uma ampla análise sócio/cultural relatando que "as pessoas trabalham muitas horas por dia e optam por fazer refeições rápidas, o aumento da renda média da população brasileira, a falta de informação: as pessoas não conhecem a cadeia alimentar, as necessidades diárias do corpo humano e as combinações saudáveis para a nossa saúde". AS e PS ressaltaram questões que envolvem diretamente a sociedade. AS destacou que a obesidade é ocasionada "em resposta a sociedade de consumo que estimula o comer e outras condutas compulsivas", e PS que "indivíduos acomodados nos confortos que a sociedade consumista oferece" acabam se tornando obesos.

Santos ${ }^{9}$ lembra que uma das explicações que podem justificar a existência do grande contingente de pessoas acima do peso no Brasil configura-se hoje como uma problemática social, podendo ainda ser entendida como uma expressão de mudanças ocorridas no mundo do trabalho, exigindo do trabalhador mais rapidez e flexibilidade inclusive nos horários e nos modos de se alimentar, das apelações exacerbadas ao consumo que leva os indivíduos a comerem e não necessariamente a se alimentarem devidamente.

A falta de tempo está presente também na questão do sedentarismo, uma vez que sem tempo as pessoas tendem a não praticar atividade física. No que se refere à atividade física, observou-se nos relatos uma séria preocupação com o aumento do sedentarismo. PT colocou que "o mundo moderno alterou o padrão de comportamento das pessoas como o aumento do sedentarismo" e, nesta mesma ideia de pensamento, NT concordou com PT, relatando que o "crescimento do sedentarismo contribui 
para a obesidade". Também com essa linha de raciocínio, MD foi bastante enfático: "o sedentarismo é também epidêmico". MC, por sua vez, destacou: a "falta de atividade física regular por preguiça ou por falta de organização do tempo" contribui para o aumento da obesidade.

Mcardle ${ }^{10}$, afirma que a dieta, por si só, não explica o significativo aumento nas taxas de obesidade em boa parte do mundo industrializado nos anos recentes. Um estilo de vida cada vez mais sedentário tem um papel significativo. Outros fatores que podem ter contribuído para esse aumento, ainda que sua ligação direta com a obesidade não seja tão bem estabelecida, são o estresse da vida moderna e o sono insuficiente.

Destacaram-se também fatores emocionais, a chamada "fome emocional", em que muitas vezes a pessoa faz uma ligação estreita entre sentimentos e a comida, come demais quando está feliz e também quando está ansioso ou triste. PT referiu que "questões de cunho psíquico/emocional e até mesmo existencial" podem influenciar na obesidade; destacando, ainda, que "a obesidade também pode ser causada por uma falta de sentido e propósito na vida". Refletindo nesse critério emocional, AS avaliou que a obesidade "pode estar vinculada a ausência de prazeres" e OS contribuiu dizendo que "indivíduos ansiosos e frustrados por não fazer parte de um padrão" evoluem para obesidade. Avaliando esse caráter emocional MD associa a obesidade a essas questões, quando relata que "alterações de comportamento alimentar, compulsão e busca de aliviar as emoções através do alimento geram o problema da obesidade no mundo moderno".

Saba ${ }^{11}$, refere que fatores psicológicos e emocionais também influenciam diretamente no acúmulo de gordura corporal, devido ao aumento na ingesta alimentar. A ansiedade e a depressão, por exemplo, costumam ser grandes incentivadores de excessos na alimentação.

\section{O tratamento da obesidade}

A pesquisa trabalhou a questão da multidisciplinaridade e da interdisciplinaridade, no tratamento da obesidade. Percebeu-se que o tratamento alinha-se à conceituação feita na primeira categoria pelos entrevistados. A obesidade é uma doença complexa e multifatorial, assim, é importante que profissionais de diferentes áreas do saber possam estar atuando de forma coordenada para que a pessoa possa obter melhores resultados; conforme destaca PS: "vejo a obesidade com causa multifatorial com consequências graves de fatores de risco a vida". PT em seu relato referiu: "o meu trabalho, deve ser parte de um programa multidisciplinar que leva em consideração o tratamento integral desta patologia, o qual inclui a atuação de vários profissionais para que o resultado seja realmente eficaz". Reforçando esse paradigma, PS relatou: "trabalho numa clínica interdisciplinar onde a obesidade é vista como um desequilíbrio no todo do indivíduo, não em suas partes ou especificidades". Nessa perspectiva de entendimento, MD afirmou que trata a obesidade "encaminhando para atividade física orientada por profissional da área, meditação, terapia psicológica e / ou Coaching". MC pontuou seu trabalho no tratamento da obesidade: "através de técnicas, modelos e ferramentas de Coaching mostramos para o cliente o que realmente é essencial em relação: a alimentação, a atividade física e ao controle de peso".

Conforme a $\mathrm{OMS}^{12}$ existem algumas recomendações para o tratamento clínico da obesidade em pessoas com Índice de Massa Corporal (IMC); acima de $30 \mathrm{~kg} / \mathrm{m} 2$ devem ser iniciadas num programa de dieta de redução calórica, exercício e outras intervenções comportamentais e estabelecer objetivos de perda de peso. Se os objetivos não forem alcançados, terapia farmacêutica pode ser oferecida. A pessoa deve ser informada da possibilidade de efeitos colaterais e da inexistência de dados sobre a segurança e eficácia de tais medicamentos no longo prazo. Pessoas com IMC acima de 40 que não alcançam seus objetivos de perda de peso (com ou sem medicamentos) e que desenvolvem outras condições derivadas da obesidade, podem receber indicação para realizarem cirurgia bariátrica. A pessoa deve ser informada dos riscos e potenciais complicações. Nesta abordagem da OMS ${ }^{12}$ percebe-se o fator interdisciplinar implicitamente necessário para o tratamento da obesidade. No aspecto avaliativo do obeso vale ressaltar que a OMS ${ }^{12}$ classifica a obesidade baseando-se no IMC definido pelo cálculo do peso corporal, em qui- 
logramas, dividido pelo quadrado da altura, em metros quadrados $\left(\mathrm{IMC}=\right.$ peso $\left./ \mathrm{h}^{2}\right)$. Analisando o aspecto alimentar no trabalho para o tratamento da obesidade AS contribui dizendo que seu trabalho "inicia pela orientação nutricional, sem restrição" (vale ressaltar aqui que AS também tem formação em nutrição, portanto tem, por lei, assegurado o direito de prescrição de dietas) e NT salienta: "elaboro um plano alimentar semanal, onde forneço opções de refeições para atingirmos o peso ideal, sempre respeitando o hábito alimentar do paciente". No que tange ao caráter nutricional do tratamento, Mahan ${ }^{13}$ alinha-se ao pensamento dos entrevistados, quando destaca que dietas que priorizam um nutriente em detrimento de outros, dietas que restringem severamente o consumo energético, bem como os jejuns prolongados, representam também um risco para a saúde.

\section{Dificuldades no tratamento da obesidade}

A pesquisa abordou dificuldades encontradas pelos entrevistados em suas práticas no trato da obesidade, destacando-se o aspecto temporal do tratamento, o aspecto comportamental e o aspecto motivacional dos obesos para manter o tratamento. Observou-se que os três pontos referidos pelos entrevistados estão diretamente relacionados. A pessoa obesa tem pressa para emagrecer, pois terá que mudar muitos hábitos e comportamentos, fazendo com que saia da sua zona de conforto; a sua motivação no início do tratamento está elevada, conforme relata AS ao dizer que os pacientes obesos estão em "busca de solução mágica" para o problema obesidade. Nesta mesma linha de raciocínio, PS contribuiu dizendo que “o processo emagrecimento nem sempre se dá na velocidade que o paciente deseja". Seguindo a mesma ideia, NT afirmou que "muitos pacientes iniciam o tratamento, mas não concluem por quererem um resultado imediato". MD relatou que "muitos (obesos) gostariam de ter um medicamento "mágico" ou "dieta milagrosa" para resolverem seu problema com a obesidade o mais rápido possível. Nesse raciocínio, MD destacou que muitos pacientes obesos "acabam realizando as cirurgias (solução mágica), o que não funciona para um grande número de operados, pois não modificaram sua mente, seu comportamento e suas emoções". Mahan ${ }^{13}$, relata, nessa perspectiva, que a procura por dietas milagrosas também tem sido um caminho buscado por uma parcela da população. Por não serem elaboradas com um cardápio balanceado, tais dietas, na maioria dos casos, promovem a perda de massa muscular e água, eletrólitos, minerais e perda de peso, porém de pouca gordura. Além disso, dietas muito restritas são de difícil adesão por um longo período. Motivados pela esperança de perda de peso em pouco tempo, muitas pessoas recorrem a estes tipos de procedimentos, muitos deles veiculados pela mídia ${ }^{13}$.

Entretanto, sabe-se que o tratamento eficaz da obesidade é lento e contínuo. Ao passar do tempo fica difícil para o obeso manter os novos hábitos saudáveis e acaba acontecendo a desmotivação e, por consequência, o abandono do tratamento, fazendo com que ocorra o aumento de peso novamente. Portanto, percebe-se que o fator motivacional é significativo para o sucesso do tratamento da obesidade. Bear ${ }^{14}$ lembra que a motivação pode ser muito abstrata ("necessidade" de passear em uma tarde de verão), mas também pode ser bastante concreta (necessidade de ir ao banheiro quando sua bexiga está cheia). PT destacou que "vícios e relação afetiva com a comida; crenças limitantes em relação à capacidade de emagrecer" dificultam o seu trabalho; e alertou: "quando a comida é relacionada com o afeto cuidado para que este afeto não seja transferido para outro meio prejudicial, como drogas e bebidas alcoólicas". AS, por sua vez, disse existir "falta de adesão por desmotivação". PS alinhou-se nesse discurso, colocando que "falta de adesão ao tratamento, recaídas quase sempre acompanhadas por episódios compulsivos, sabotagens, efeito rebote devido a culpa" dificultam seu trabalho. PS também destacou: "a resistência que o obeso apresenta de 'se ver', 'se olhar' por dentro é uma das principais dificuldades". MC ressaltou "a importância de associar o seu tratamento a algo de real importância para a pessoa, no caso os seus valores e crenças".

Gazzaniga ${ }^{15}$ relaciona a motivação diretamente às questões do tratamento da obesidade, ao orientar que a motivação ativa, dirige e sustenta a ação através de quatro qualidades essenciais dos estados motivacionais:

Estados motivacionais energizantes: ativam ou 
estimulam comportamentos; por exemplo, o desejo de estar fisicamente em forma faz com que a pessoa saia da cama em uma manhã fria para praticar exercícios físicos.

Estados motivacionais diretivos: orientam comportamentos para satisfazer objetivos ou necessidades específicas; por exemplo, a fome motiva a comer, a sede motiva a beber.

Estados motivacionais de persistência: ajudam a pessoa a persistir no seu comportamento até que seu objetivo seja atingido. Exemplo é o caso de a fome nos atormentar até encontrarmos algo para comer; a persistência nos leva a fazer a escolha correta do alimento.

Estados motivacionais de motivo: os motivos diferem em sua força, dependendo de fatores internos e externos, e normalmente nos deparamos com motivos concorrentes. Por exemplo: devemos comer pizza (prazer) ou carboidratos integrais (saúde/emagrecimento)? O motivo mais forte, normalmente, vence.

\section{O coaching no tratamento da obesidade}

Para o desenvolvimento deste tema fez-se necessário uma conceituação de coaching de emagrecimento, muito relacionado com a ideia de a mudança comportamental ser determinante para o sucesso do tratamento.

Observou-se que o coaching pode ser um grande aliado para o tratamento da obesidade, ao fazer parte de um programa integrado de forma multidisciplinar e atuar fortemente na mudança comportamental necessária para o sucesso do tratamento, principalmente para manter o indivíduo motivado atuando diretamente em questões emocionais. Avaliando o aspecto comportamental, os entrevistados contribuíram no sentido de acreditarem que o coaching promove mudanças comportamentais, como mostra o relato de PT: "coaching de emagrecimento gera mudanças: física e mental; esta última fundamental para a sustentação do estilo de vida saudável e equilíbrio emocional".

Dentro dessa análise o fator foco também é muito importante. Na percepção de AS, "são muitos atravessamentos que ocorrem na relação com o peso, o comer e a obesidade, o coaching ajuda a manter o foco para o objetivo proposto". NT entende que "com o coaching o paciente consegue perceber seu real objetivo com o tratamento e traçar metas para alcançá-lo". Nessa mesma linha de pensamento relacionada a mudanças comportamentais, MD afirmou que o coaching "promove mudança dos hábitos alimentares, estilo de vida e até mesmo em questões mais profundas na vida dos coachees".

A obesidade é uma das questões centrais da saúde pública no cenário contemporâneo, principalmente nas sociedades industrializadas. Isso já seria um bom motivo para que ciências comportamentais se dedicassem ao tratamento do excesso de peso; e ainda é necessário considerar que a obesidade pode afetar a autoestima das pessoas, impedindo-as de estabelecer vínculos e desfrutar de relações saudáveis, além de ser um estorvo em muitos setores da vida, inclusive na sexual e profissional ${ }^{16}$.

Nos dias de hoje dispomos de modernas e avançadas técnicas de reprogramação mental, já usadas consagradamente em vários setores, que podem também contribuir substancialmente para a cura da obesidade, já que esta é uma doença com consequências e desdobramentos nefastos ${ }^{17}$. Nos casos não clínicos, ou seja, naqueles em que obesidade instala-se sem que existam condições de mau funcionamento fisiológico e orgânico, ela encontra-se inexoravelmente associada às causas emocionais, como a compulsão alimentar, ansiedade, depressão, baixa autoestima, padrões familiares e sistemas de crenças e identidade que levam o indivíduo ao excesso de peso ${ }^{16}$. PS avaliou: "na maior parte dos casos vejo que obesidade tem vínculo profundo com a história de vida pregressa". MD, por sua vez, afirmou que o coaching promove "ampliação da consciência de vida, nos comportamentos, emoções e tudo que vinha causando uma alimentação errônea e consequente ganho de peso". MC, traduz a efetividade do coaching dizendo que "o coaching permite que o cliente consiga visualizar através de perguntas poderosas, as verdadeiras respostas que o fazem querer se livrar do excesso de peso e querer ter uma vida saudável, longeva e feliz".

Segundo visto em Carvalho ${ }^{7}$, o coaching pode ser um aliado de pessoas que desejam perder ou manter o peso, pois conduz à mudanças de hábitos sabotadores que as impedem de levar uma vida saudável, 
e a adquirir comportamentos que auxiliarão na redução e manutenção de peso.

\section{A aplicação da metodologia coaching no tratamento da obesidade}

A pesquisa estudou como é feita a aplicação da metodologia coaching no tratamento da obesidade, destacando o aspecto avaliativo comportamental, a gestão e o planejamento, o aspecto motivacional e o método coaching através de técnicas e ferramentas. O primeiro ponto que discorreu foi sobre o aspecto avaliativo comportamental, que traduz-se pelo direcionamento do Coach para que a pessoa obesa consiga identificar o seu estado atual e como ele chegou nesse estado, buscando identificar possíveis causas da sua obesidade, padrões de comportamento não saudáveis, comportamentos sabotadores e crenças limitantes. Para PT "o Coaching de Emagrecimento identifica as possíveis causas da obesidade, identifica os padrões de comportamento não saudáveis, os comportamentos sabotadores, as crenças limitantes por meio de uma avaliação dos quatro quadrantes do ser, baseada na Teoria Integral de Ken Wilber". AS relatou: "penso que auxilia em muito, pois direciona o foco". Nessa contribuição comportamental do coaching, PT destacou sua aplicação do coaching para "tomada de consciência, senso de responsabilidade pela própria saúde, foco e disciplina, são aspectos trabalhados no programa que fortalecem as mudanças de hábitos".

Após avaliar esses aspectos, pode-se passar para o segundo passo identificado pelos entrevistados que foi o planejamento para traçar um plano de ação eficaz para um emagrecimento sustentável, o qual estabelece metas e objetivos bem definidos. PT afirmou que "o programa ajuda o indivíduo a traçar um plano de ação eficaz para um emagrecimento sustentável, o qual estabelece metas e objetivos". PS também mencionou o quesito planejamento: "trabaIho com um vínculo estreito com a nutricionista da equipe, peço auxílio a ela para que trabalhemos juntas as metas com relação ao peso a ser atingido". PT ressaltou: "indivíduos obesos que não sabem se organizar para iniciar o tratamento. O coaching contribui com a gestão eficaz da saúde e dá suporte ao indivíduo no seu processo de emagrecimento".
Nesse momento será importante o aspecto motivacional para seguir o planejamento. PT avaliou que o coaching "desenvolve as capacidades e habilidades que cada indivíduo precisa para uma mudança no estilo de vida". AS, por sua vez, relatou que o coaching "possibilita o desmonte de crenças e desculpas/justificativas que o dependente a alimento tende a usar justificando sua conduta e seu enfrentamento". PS destacou sua preocupação com o aspecto motivacional "a meta deve ser real e em períodos de tempo pequenos (quinze dias, 1 mês) para não desmotivar". Na mesma linha de proposta motivacional, NT afirmou: "utilizo ferramentas poderosas que instigam o paciente a buscar seu objetivo com motivação". O coaching desenvolve capacidades e habilidades que cada pessoa precisará para uma mudança no seu estilo de vida atual. Tudo isso é feito através de técnicas e ferramentas que norteiam o coach para que este conduza um processo de coaching eficaz, motivando a pessoa obesa a alcançar o emagrecimento desejado.

Observando um método coaching para emagrecimento, Volpato ${ }^{17}$ relata que através de técnicas oriundas da Programação Neuro Linguística e da hipnose, entre outras, no processo de coaching aplicado à desestruturação da obesidade, o coach poderá identificar e alterar raízes emocionais que inconscientemente levam ao aumento de peso, ou alimentam um processo de dependência ao ato de comer e, o mais importante de tudo, poderá compreender e modificar uma estrutura emocional que impede de emagrecer.

\section{CONCLUSÃo}

Estudando possíveis estratégias da metodologia em tela para o tratamento da obesidade, observou-se que o coaching pode ser um grande aliado para o tratamento da obesidade, fazendo parte de um programa integrado multidisciplinar e atuando fortemente na mudança comportamental necessária para o sucesso do tratamento e, principalmente, para manter o indivíduo motivado ao atuar diretamente em questões emocionais. Analisando como é feita aplicação de metodologia coaching no tratamento da obesidade, pelos profissionais da saúde entrevis- 
tados pela pesquisa, destacou-se o aspecto avaliativo comportamental, a gestão e planejamento, o aspecto motivacional e o método coaching através de técnicas e ferramentas. Assim, constatou-se que, para que o tratamento da obesidade tenha sucesso, motivação, planejamento e disciplina são fatores relevantes e com o auxílio do coaching a eficácia desses fatores poderá ser maior.

Através do coaching a pessoa obesa poderá ter estratégias importantes para trabalhar sua autodeterminação e motivação, aumentando, assim, seu esforço para que não haver desistências diante dos desafios. A autoeficácia também poderá ser atingida por meio de técnicas correlatas, aumentando crenças fortalecedoras, autoestima e autoaceitação; tornando possível a mudança de comportamentos de pessoas obesas para hábitos mais saudáveis e produtivos e contribuindo na evolução do emagrecimento durante o processo.

$\mathrm{O}$ aumento de peso pode estar diretamente ligado a problemas emocionais. O coaching poderá ajudar pessoas a identificar o que está gerando em si mesmas tal comportamento e, a partir da aplicação de técnicas para controle do estado emocional, colaborar no desenvolvimento de estratégias significativas. Porém, mudanças de hábitos podem também gerar desistência. Para que isso não ocorra, o coaching precisará associar as metas e os objetivos aos valores, alinhando a mudança a um propósito maior e ajudando as pessoas obesas a compreenderem que emagrecer saudavelmente e com sentido poderá proporcionar-lhes bem-estar e qualidade de vida.

\section{REFERÊNCIAS}

1 ORGANIZAÇÃO MUNDIAL DA SAÚDE. Estatísticas mundiais de saúde 2006. OMS, 2006.

2 INSTITUTO BRASILEIRO DE GEOGRAFIA E ESTATÍSTICA. Levantamento Antropometria - Estado Nutricional de Crianças e Adultos no Brasil - da Pesquisa de Orçamentos Familiares (POF) 2008-2009. IBGE, Rio de Janeiro, 2010.

3 ORGANIZAÇÃO MUNDIAL DA SAÚDE. Estatísticas mundiais de saúde 2012. OMS, 2012.

4 SABA, Fabio. Mexa-se: atividade física, saúde e bem-estar. 3. ed. São Paulo: Phorte, 2011.
5 BOAS, L. C. G. V.; FOSS, M. C.; FREITAS, M. C. F.; TORRES, H. C.; MONTEIRO, L. Z.; PACE, A. E. Adesão à dieta e ao exercício físico das pessoas com diabetes mellitus. Texto Contexto Enfermagem, Florianópolis, 2011.

6 PAIVA, Luiz A.; MANCILHA, Jairo; RICHARDS, Jonh. Coaching: passo a passo. Rio de Janeiro: Qualitymark Editora, 2012.

7 CARVALHO, Denis. Coaching de emagrecimento. Coordenação Editorial: PERCIA, André; MATTEU, Douglas; MARQUES, José R.; SITA, Mauricio. Master Coaches - Técnicas e relatos de mestres do coaching. São Paulo: Editora Ser Mais, 2012.

8 BARDIN, L.. Análise de conteúdo. São Paulo: Edições 70, 2011.

9 SANTOS, Milton. Por uma outra globalização: do pensamento único à consciência universal. 10. ed. Rio de Janeiro: Record, 2003.

10 MCARDLE, Willians D.; KATCH, Frank I.; KATCH, Vitor L. Nutrição para o esporte e o exercício. 3. ed. Rio de Janeiro: Guanabara Koogan, 2011.

11 SABA, Fabio. Aderência: a prática do exercício físico em academias. Barueri: Manole, 2001.

12 ORGANIZAÇÃO MUNDIAL DA SAÚDE. Estatísticas mundiais de saúde 2011. OMS, 2011.

13 MAHAN, Kathleen L.; STUMP-ESCOTT, Silvia. Krause, alimentos, nutrição e dietoterapia. 12. ed. Rio de Janeiro: Elsevier, 2010.

14 BEAR, M. F.; CONNORS, B. W.; PARADISO, M. A. Neurociências desvendando o sistema nervoso. 3. ed. Porto Alegre: Artmed, 2008.

15 GAZZANIGA, Michael S.; HEATHERTON, Todd F. Ciência psicológica - mente, cérebro e comportamento. Porto Alegre: Artmed, 2005.

16 DEBSKI, Roberto. Coaching de emagrecimento: conquiste a leveza e a saúde com que sempre sonhou. Coordenação Editorial: PERCIA, André; SITA, Mauricio. Coaching - Grandes mestres ensinam como estabelecer e alcançar resultados extraordinários na sua vida pessoal e profissional. São Paulo: Editora Ser Mais, 2013.

17 VOLPATO, Ana Maria. Coaching de Saúde: o atendimento que você sempre quis. Coordenação Editorial PERCIA, André; MATTEU, Douglas; MARQUES, José R.; SITA, Mauricio. Master Coaches - Técnicas e relatos de mestres do coaching. São Paulo: Editora Ser Mais, 2012. 